\title{
Cardiovascular disease determinants: Burden and knowledge among women in a rural community of Delhi
}

\author{
Jitendra K Meena, Anjana Verma, Bratati Banerjee, Gopal Krishna Ingle \\ Department of Community Medicine, Maulana Azad Medical College, New Delhi, India. \\ Correspondence to: Jitendra K Meena, E-mail: drmeenajk@gmail.com
}

Received September 30, 2014. Accepted October 21, 2014

\section{Abstract}

Background: Cardiovascular diseases (CVDs) are becoming major health burden in developing countries such as India. Rural women are reported to have high CVD-related mortality rates and are less likely to receive appropriate treatment.

Objective: To assess knowledge, practices, and burden of CVD-related risk factors among women in a rural area of Delhi.

Materials and Methods: A cross-sectional study was conducted at Pooth Khurd village, a rural pocket situated in northwest district of Delhi through house-to-house survey by two investigators. A total of 100 women above 18 years of age and resident of the village were included in the study using systematic random sampling technique. A pretested interview schedule, containing items to assess knowledge and practices regarding CVDs and risk factors based on various validated tools, was used. Fischer's exact and Pearson's correlation tests were applied; tests were two sided with $p$-value below 0.05 considered significant.

Results: Poor CVD knowledge was seen among study participants with less than half of subjects identifying family history $(24 \%)$, tobacco and alcohol intake $(48 \%, 47 \%)$, and so forth as risk factors for CVDs. High burden of risk factors, such as high salt intake (35\%), inadequate physical activity (77\%), and raised blood sugar (5\%), and poor health-seeking behavior were observed.

Conclusion: This study reflects poor knowledge and relatively high burden of CVD risk factors among rural women. Poor knowledge is predictive of high CVD risk in community settings.

KEY WORDS: Rural, women, cardiovascular risk factors, urbanization, knowledge

\section{Introduction}

The prevalence of cardiovascular disease (CVD) is rising worldwide and it accounts for $17 \%$ of the total mortality. This escalation in the prevalence of CVD has been attributed to the paradigm shift in lifestyle including the changes in dietary pattern, particularly more consumption of refined carbohydrates and saturated lipids, and physical inertia associated with progressive economic growth and urbanization. ${ }^{[1]}$ Among Indians, CVD tend to occur more and earlier in life than among any other ethnic group. ${ }^{[2]}$ Global Burden of Disease Study has reported that ischemic heart disease and stroke are

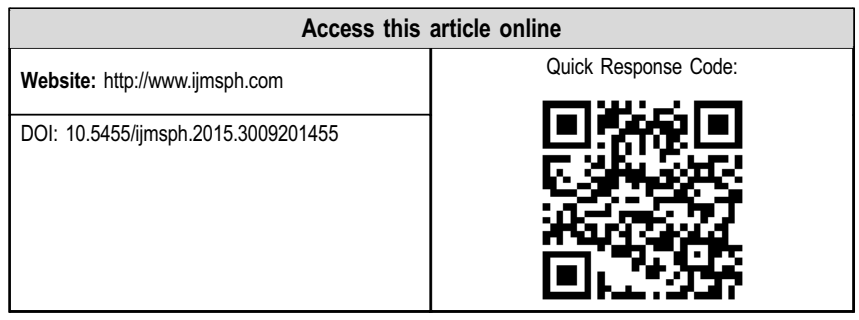

the most important causes of years of life lost, disability, and mortality in women. ${ }^{[3]}$ The report on causes of death by the Registrar General of India also reported CVDs as the most important cause of mortality in women. ${ }^{[4]}$ Among women, metabolic risk factors such as dyslipidemia, hypertension, diabetes, and high waist-to-hip ratio are particularly more important. [5]

The Second and Third National Family Health Surveys (NFHS) reported that smokeless tobacco use was particularly high in Indian women and was increasing among women, more among the illiterate and those with low educational and socioeconomic status (SES). ${ }^{[6]}$ The NFHS also reported a greater prevalence of obesity in Indian women as compared to men with an increasing trend to overweight and obesity. ${ }^{[7]}$ Studies from different regions of the country have reported a high prevalence of hypertension, metabolic syndrome, diabetes, and other cardiometabolic risk factors more prevalent in middleaged and older women as compared to men. ${ }^{[8,9]}$

Indian women have high CVD-related mortality rates and are less likely to receive appropriate treatment as compared to men owing to poor knowledge regarding disease, risk factors, preventive strategies, and various social factors. Women in 
rural India have lower social status (except in a few states), more episodes of illness than men, and also are less likely to access health-care facilities before the illness is well advanced. ${ }^{[10]}$ Though prevalence of CVDs is lower in rural population, it is believed to increase owing to rapid urbanization and changing lifestyle patterns.

\section{Materials and Methods}

A population-based, cross-sectional survey was conducted in Pooth Khurd village. Study sample size calculated by estimating knowledge about CVDs among women to be $55.6 \%$ based on previous study ${ }^{[11]}$ with relative error of $20 \%$ was 83. However, final sample size of 100 women above 18 years of age residing in the village was used considering a nonresponse rate of $10 \%$. The study was divided into two groups: (1) survey for assessing knowledge (self-reported) and (2) survey for prevalence assessment of various factors/ practices regarding CVDs and its risk factors.

The study was conducted for 1 month (March 2014) using a predesigned, pretested, semi-structured interview schedule, containing items to assess demographic information, knowledge and practices, and prevalence of various CVDs (such as hypertension and diabetes) and their various risk factors (such as smoking, obesity, diabetes, dietary habits, mental stress, and physical inactivity). The Cronbach's alpha coefficient was calculated to assess internal consistencies of questionnaire and was found to be 0.768 .

Systematic random sampling technique was adopted for selection of study participants. Total numbers of houses in the village were 576 and therefore every fifth house was selected for the study. One woman each was randomly chosen from selected houses and those meeting the criteria (above 18 years) were included in the study. In case of nonavailability, eligible woman from next selected house was included. Subjects were interviewed by investigators comprising of one male and one female resident doctors (Community Medicine) assisted by local female village health workers (AWW, ASHA, etc). Health workers were explained the purpose of the study and were requested to participate and assist in the data collection and verification if needed on a voluntary basis. Interview was conducted in Hindi, which is spoken and understood well in the area of study. Interview schedule comprised questions to assess demographic details: name, age, marital status, literacy status, monthly income, types of family system and number of family members, and SES, defined as per modified Prasad's classification. ${ }^{[12]}$

CVD was defined as a disease of the heart or blood vessels. Subjects were assessed based on CVD history (self-reported), knowledge or practices regarding CVD risk factors such as old age, overweight, family history of CVD, inadequate physical activity, tobacco intake, smoking, alcohol, high salt intake, inadequate fruit/vegetable intake, high lipids intake, mental stress, raised blood sugar, raised blood pressure, and sleep disorders. Study questionnaire was adapted from various validated tools for accurate assessment of CVD risk factors, namely the WHO STEPwise Approach Questionnaire v.2.1, ${ }^{[13]}$ Goldberg General Health Questionnaire (GHQ-12), ${ }^{[14]}$ and Berlin Questionnaire. ${ }^{[15]}$ Study participants were also assessed for blood pressure/sugar/lipid checkup in the past 1 year and treatment-seeking behavior.

The objectives of the study were explained to study participants and informed consent was obtained and confidentiality of data was ensured. Interview sheets were duly filled by investigator and data were fed in MS Excel and were analyzed using Statistical Package for the Social Sciences software, version 16.0 (SPSS Inc., Chicago, IL). Descriptive statistics, including mean, standard deviation, and range, for quantitative data and proportions for qualitative data were used to characterize the study population. $\chi^{2}$-Test or Fischer's exact test was used to observe differences between proportions for independent groups. Pearson's correlation test was applied to determine relationship between two variables. All statistical tests were two sided and performed at a significance of $\alpha=0.05$.

\section{Results}

Mean age of participants was 35.2 years and majority $88 \%$ of them married. Almost half of the participants $(47 \%)$ were illiterate and their average per capita monthly income was Rs. $1634.9 \pm 884.6$. Most of the households were joint families $(72 \%)$ and average household strength was 6.8 people. Significant number of participants $(70 \%)$ belonged to lower SES (grades IV and V) based on monthly income.

Table and Figure 1 show knowledge and prevalence of CVD risk factors. Study participants were asked if the factor in the question increased the risk of developing CVD. The agreement ranged from $24 \%$ for family history to $81 \%$ for inadequate fruit/vegetable intake. On relationship analysis between knowledge and presence of CVD risk factors it was seen that poor knowledge of various risk factors (such as overweight, tobacco intake, smoking, high salt consumption, sleep disorders, mental stress, and high blood sugar) has increased risk for development of said risk factors.

Among lifestyle factors tobacco intake was seen among $21(21 \%)$ participants, though $8(38 \%)$ participants wanted to quit it. Consumption of alcohol was reported by three respondents, of which one was willing to quit. Sleep problems were present among $17 \%$ participants, of which only $3(17.6 \%)$ participants took treatment. Snoring was present among $29 \%$ respondents, and $15 \%$ participants had family history of CVD. In the study, $45 \%$ respondents were found to be mentally stressed and when assessed about stress coping mechanism mostly $31(68.8 \%)$ did nothing, $10(22.2 \%)$ talk and discuss with peers/family, $3(0.6 \%)$ took treatment, and $2(0.4 \%)$ did yoga/meditation. Married women were found to be more ignorant about mental stress and did nothing about it. Around one-fourth (22\%) participants believed that they were overweight, mostly $10(45.4 \%)$ subjects did nothing while some 


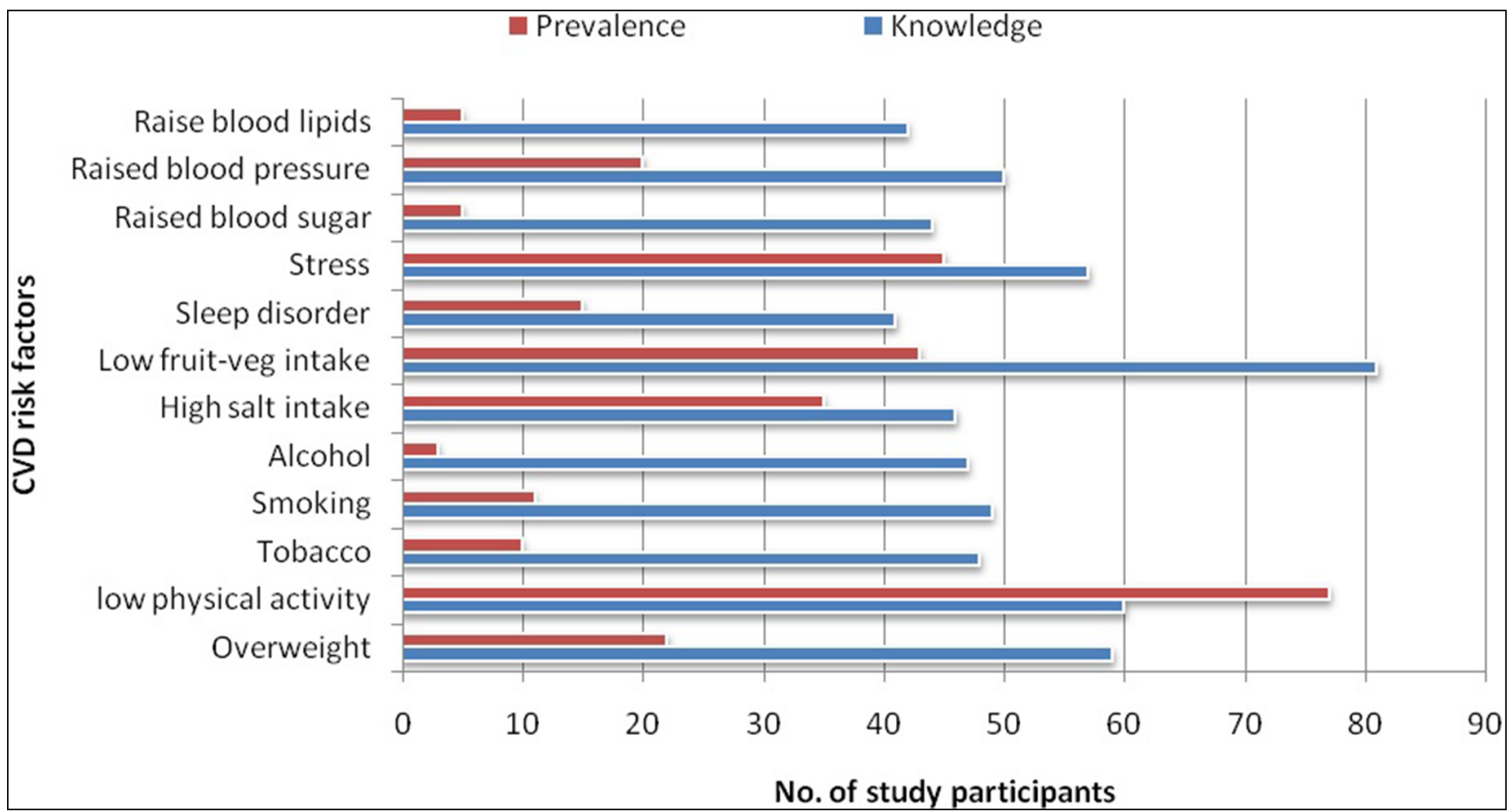

Figure 1: Knowledge and prevalence of various cardiovascular risk factors among study participants.

practiced exercise $6(27.2 \%)$ [diet reduction (13.6\%) and skipping meals $(13.6 \%)]$ for weight reduction. Sedentary lifestyle was common as $77 \%$ participants reported inadequate physical activity.

Dietary risk factors were assessed and it was seen that only $56 \%$ respondents were eating adequate fruits and/or vegetables ( $>5$ servings per day). High salt intake was noticed among $35 \%$ study participants, among whom only 4 (11.4\%) participants had tried reduction. Oil consumption types and preferences were also assessed, $69 \%$ respondents were using mustard oil, $27 \%$ refined oil, and $4 \%$ ghee for cooking purpose.

Table 1: Prevalence and association between knowledge of cardiovascular disease risk factors among participants $(n=100)$

\begin{tabular}{lccc}
\hline Risk factor & Knowledge (\%) & Prevalence (\%) & $\chi^{2}-, p$-, \& $\boldsymbol{R}$-value \\
\hline Nonmodifiable & & & - \\
$\quad$ Higher age & 43 & - & - \\
$\quad$ Family history & 24 & 15 & $0.3,0.8,-0.01$ \\
Modifiable & & & $12.1,<0.1^{*}, 0.34$ \\
$\quad$ Overweight & 59 & 22 & $1.4,0.09,-0.12$ \\
Inadequate physical activity & 60 & 77 & $0.06,0.8,-0.02$ \\
Tobacco & 48 & 10 & $0.4,0.4,0.6$ \\
Smoking & 49 & 11 & $4.6,0.03^{*},-0.21$ \\
Alcohol & 47 & 3 & $5.0,0.08,0.22$ \\
High salt intake & 46 & 35 & $1.1,0.2,-0.10$ \\
Inadequate fruit/vegetable intake & 81 & 43 & $2.2,0.1,-0.14$ \\
Sleep disorder & 41 & 15 & $1.3,0.2,-0.11$ \\
Stress & 57 & 45 & $0.16,0.68,0.04$ \\
Raised blood sugar & 44 & 5 & $3.1,0.07,0.1$ \\
Raised blood pressure & 50 & 20 & 5 \\
Raise blood lipids & 42 & & \\
\hline
\end{tabular}

Fischer's exact $\left(\chi^{2}\right)$ and Pearson's correlation $(R)$ tests were applied. ${ }^{*} p<0.05$ was considered significant. 
Table 2: Knowledge and predictors of CVD risk factors among study participants

\begin{tabular}{|c|c|c|}
\hline Knowledge & Predictors & $\chi^{2} \& p$-value ${ }^{*}$ \\
\hline Higher age & High age ( $>42$ years) & $14.1,0.004$ \\
\hline Inadequate fruit and vegetable intake & & $14.2,0.002$ \\
\hline Overweight & & $11.2,0.02$ \\
\hline Inadequate physical activity & & $10.7,0.03$ \\
\hline Smoking & & $8.7,0.05$ \\
\hline Raised blood sugar & Higher education (intermediate or above) & $16.5,0.02$ \\
\hline Raised blood sugar & Joint family & $4.8,0.02$ \\
\hline
\end{tabular}

SES, socioeconomic status.

Fischer's exact $\left(\chi^{2}\right)$ test was applied.

*Only significant associations $(p<0.05)$ are listed.

Table 3: Predictors of CVD risk factors among study participants

\begin{tabular}{|c|c|c|}
\hline Risk factor & Predictors & $\chi^{2} \& p$-value ${ }^{*}$ \\
\hline Salt intake & Marital status (single) & $7.9,0.013$ \\
\hline \multirow[t]{2}{*}{ Inadequate physical activity } & Marital status (married) & $8.2,0.013$ \\
\hline & Lower SES (grades IV \& V) & $14.8,0.003$ \\
\hline Tobacco intake & Higher age group ( $>42$ years) & $14.1,0.003$ \\
\hline Smoking & & $12.6,0.007$ \\
\hline Sleep disorders & & $25.4,<0.01$ \\
\hline Raised blood pressure & & $9.1,0.05$ \\
\hline Raised blood sugar & & $11.8,0.006$ \\
\hline Raised blood lipids & Upper SES (grades I \& II) & $18.4,0.006$ \\
\hline Sleep disorders & & $9.0,0.04$ \\
\hline
\end{tabular}

SES, socioeconomic status.

Fischer's exact $\left(\chi^{2}\right)$ test was applied.

*Only significant associations $(p<0.05)$ are listed.

Table 2 shows predictors for knowledge of CVD risk factors among study participants. Higher age of the participants was found significantly associated with their knowledge about old age and inadequate fruits/vegetables intake as risk factors of CVD. Better SES was associated with good knowledge of several risk factors such as family history, overweight, inadequate physical activity, smoking, raised blood sugar and pressure. Participants with better educational status or belonging to joint families had better knowledge of raised blood sugar as a CVD risk factor.

Table 3 shows the predictors of CVD risk factors among study participants. Participants from lower SES were inadequately physically active but oil consumption and sleep disorders were found significantly higher among those with better SES. Tobacco consumption (chewable/smoking) was found to be significantly higher among aged participants (>42 years). In contrast, younger participants were found more willing to quit tobacco consumption. High salt intake was seen among single women and married participants were mostly sedentary with inadequate physical activity. Raised blood pressure was significantly associated with higher age (>42 years) and its treatment-seeking behavior was significantly high in participants with better education and SES. Raised blood sugar and its treatment-seeking behavior was high among higher aged ( $>40$ years) participants.

Table 4 shows health-seeking behavior among participants. It was seen that $51 \%$ and $26 \%$ participants, respectively, had got their blood pressure and blood sugar checked in past 1 year. Similarly, prevalence of high blood pressure and high blood sugar was $19 \%$ and $5 \%$ and $6(31.5 \%)$ and $4(80 \%)$

Table 4: Health-seeking behavior among study participants $(n=100)$

\begin{tabular}{lcc}
\hline & Blood pressure (\%) & Blood sugar (\%) \\
\hline Checkup (1 year) & 51 & 26 \\
Raised BP/sugar & 19 & 5 \\
Treatment taken & 6 & 4 \\
\hline
\end{tabular}

$\mathrm{BP}$, blood pressure. 
participants took antihypertensive and antidiabetic treatment, respectively. Study participants were assessed for general morbidity profile and treatment-seeking behavior. It was found that 13 participants had various cardiovascular and related illness (including hypertension, diabetes, and stroke); of which, only $9(69.2 \%)$ participants took any kind of treatment, of which mostly $(8 \%)$ preferred allopathic treatment and only half $(4 \%)$ of them reported taking it regularly. When enquired, $16 \%$ participants reported having blood cholesterol tested in past 1 year and of them, $5(31.2 \%)$ had high blood cholesterol for which $3(60 \%)$ reduced their oil consumption, $1(20 \%)$ took drug-based treatment, and $1(20 \%)$ did nothing.

\section{Discussion}

We included women as young as 18 years based on the notion that CVD risk factors should be screened and identified at an earlier age, so that preventative behaviors can be established to prevent the onset and prevalence of CVD. Majority of the study participants were married $(88 \%)$ belonging to lower SES $(70 \%)$ hailing from joint families $(72 \%)$, which are common characteristics of women in such rural settings.

It was observed that average half of the women enrolled in the study had knowledge about one or other CVD risk factors. These findings are similar to a study conducted by Yahya et al. ${ }^{[11]}$ among rural women in Malaysia where knowledge prevalence was $55.6 \%$. Interestingly, it was also seen that as high as $81 \%$ study participants identified inadequate fruit/ vegetable intake as risk factor, which could be a general notion that fruits and vegetables are health-improving foods may not specifically as cardioprotective. It was found that participants with higher SES had significantly higher oil consumption, raised blood lipid levels, and sleep disorders despite having significantly better knowledge of other CVD risk factors such as overweight, smoking, inadequate fruit/vegetable intake, and raised blood pressure. Participants with better educational status and had better awareness and sought treatment of raised blood pressure. Similar findings were reported by Sadhukhan and Khan ${ }^{[16]}$ in a study carried out among adults in a rural community of Bengal where CVD risk was significantly contributed by age, education, and economic status of the study subjects.

In a similar study conducted by Pandey et al. ${ }^{[17]}$ to assess CVD-related knowledge and behaviors in Asian Indian women, the prevalence of chronic risk factors in rural women were smoking/tobacco use $(39.3 \%)$, high salt intake $(18.2 \%)$, low physical activity (59.5\%), overweight/obesity (22.5\%), hypertension (31.6\%), and diabetes (4.3\%). Our study presents similar findings though comparatively lower for smoking and hypertension but burden of other risk factors such as high salt intake (35\%), inadequate physical activity $(77 \%)$, and raised blood sugar $(5 \%)$ was found higher in our study. However, the prevalence of overweight $(22 \%)$ was almost similar. The WHO-ICMR NCD risk factor surveillance ${ }^{[18]}$ conducted among 13,527 rural residents reported obesity
$(29.6 \%)$, inadequate physical activity $(35 \%)$, self-reported diabetes and hypertension (3.1, 7.2\%), and nil prevalence of smoking among women, indicating geographical and lifestyle differences for variability in risk factors. Poor health-seeking behavior was seen among subjects with only half $(51 \%)$ had got blood pressure checked in past 1 year and even poorer picture for blood sugar testing (26\%) and lipid levels testing $(16 \%)$. Apart from knowledge, treatment-seeking behavior is influenced by various other factors such as prevailing attitudes, availability of health services, and its cost, which requires further debate.

We observed an inverse relationship of certain CVD risk factors with their knowledge among participants-overweight, tobacco intake, high salt intake, sleep disorder, mental stress, and raised blood pressure. However, association was found low and not significant, which could be due to limited scope of the study. But it certainly shows that poor knowledge is indicative of high CVD risk factor burden among study participants. Unifocal urbanization without adaption of healthy lifestyle in rural areas characterized by a marked increase in the intake of energy-dense foods, a decrease in physical activity, and a heightened level of psychosocial stress will subsequently promote the development of dysglycemia, hypertension, and dyslipidemia. ${ }^{[19]}$ Therefore, it is recommended that resources should be directed toward applying the existing knowledge base to tackle the CVD epidemic in policy, capacity building, and research arenas.

This research is per knowledge first of its kind conducted in north India carried out exclusively among rural women paving way for further research and interventions in this group. Focus of study on women only may be considered a limitation, but we consider this as study strength because influencing behavior of women has transformative benefits on the society as suggested by the WHO. ${ }^{[20]}$ The study was resource limited and lacked investigations (anthropometric and clinical, biochemical investigations, and qualitative research), which could have provided better estimation prevalence of various CVD risk factors. The study has been performed at a single site in India and may not represent the whole country.

\section{Conclusions}

This study reflects poor knowledge and relatively high burden of CVD risk factors among rural women. Poor knowledge is predictive of high CVD risk in community settings.

\section{References}

1. Pagidipati NJ, Gaziano TA. Review Estimating deaths from cardiovascular disease: a review of global methodologies of mortality measurement. Circulation 2013;127(6):749-56.

2. Goyal A, Yusuf S. The burden of cardiovascular disease in the Indian subcontinent. Indian J Med Res 2006;124(3): 235-44.

3. Lozano R, Naghavi M, Foreman K, Lim S, Shibuya K, Aboyans V, et al. Global and regional mortality from 235 causes of death for 
20 age-groups in 1990 and 2010: a systematic analysis for the Global Burden of Disease Study 2010. Lancet 2012;380: 2095-128.

4. Office of Registrar General. Report on Causes of Death in India 2001-2003. New Delhi: Office of Registrar General, Ministry of Home Affairs, Government of India, India, 2009.

5. Anand SS, Islam S, Rosengren A, Franzosi MG, Steyn K, Yusufali $\mathrm{AH}$, et al. Risk factors for myocardial infarction in women and men: insights from the INTERHEART study. Eur Heart $\mathrm{J}$ 2008;29:932-40.

6. Bhan N, Srivastava S, Agrawal S, Subramanyam M, Millet C, Selvaraj S, et al. Are socioeconomic disparities in tobacco consumption increasing in India? A repeated cross-sectional multilevel analysis. BMJ Open 2012;2:e001348.

7. Wang $\mathrm{Y}$, Chen HJ, Shaikh S, Mathur P. Is obesity becoming a public health problem in India? Examine the shift from under- to overnutrition problems over time. Obes Rev 2009;10:456-74.

8. Gupta R, Guptha S, Sharma KK, Gupta A, Deedwania PC. Regional variations in cardiovascular risk factors in India: India heart watch. World J Cardiol 2012;4:112-20.

9. Chopra SM, Misra A, Gulati S, Gupta R. Overweight, obesity and related non-communicable diseases in Asian Indian girls and women. Eur J Clin Nutr 2013;67:688-96.

10. United Nations Population Fund. The State of World population: 1997-The Right to Choose: Reproductive Rights and Reproductive Health. , New York: UNFPA, 1997.

11. Yahya R, Muhamad R, Yusoff HM. Association between knowledge, attitude and practice on cardiovascular disease among women in Kelantan, Malaysia. Int J Collab Res Intern Med Public Health 2012;4:1507-23.

12. Sharma R. Revision of Prasad's social classification and provision of an online tool for real-time updating. South Asian J Cancer 2013;2(3):157.

13. World Health Organization. The WHO STEPwise Approach to Chronic Disease Risk Factor Surveillance (STEPS) Instrument v-2.1 (Core and Expanded), 2012. Available athttp://www.who.int/ chp/steps/STEPS Instrument v2.1.pdf (last accessed on September 15, 2012).

14. Goldberg D, Williams P. A User's Guide to the General Health Questionnaire. Basingstoke: NFER-Nelson, 1988. pp 3-4.

15. Chung F. Validation of the Berlin questionnaire and American Society of Anesthesiologists checklist as screening tools for obstructive sleep apnea in surgical patients. Anesthesiology 2008;108:822-30.

16. Sadhukhan SK, Khan S. Blood pressure awareness among general population: a rural west Bengal experience with logistic regression. Natl J Med Res 2012;2(1):55-8.

17. Pandey RM, Agrawal A, Misra A, Vikram NK, Misra P, Dey S, et al. Population-based intervention for cardiovascular diseases related knowledge and behaviours in Asian Indian women. Indian Heart J 2013;65(1):40-7.

18. Mohan V, Mathur P, Deepa R, Deepa M, Shukla DK, Menon GR, et al. Urban rural differences in prevalence of self-reported diabetes in India-the WHO-ICMR Indian NCD risk factor surveillance. Diabetes Res Clin Pract 2008;80:159-68.

19. Yusuf S, Reddy S, Ounpuu S, Anand S. Global burden of cardiovascular diseases: part I: general considerations, the epidemiologic transition, risk factors, and impact of urbanization. Circulation 2001;104:2746-53.

20. World Health Organization. Diet, Nutrition and the Prevention of Chronic Diseases. Report of a Joint WHO-FAO Consultation. Geneva: World Health Organization, 2003

How to cite this article: Meena JK, Verma A, Banerjee B, Ingle GK. Cardiovascular disease determinants: Burden and knowledge among women in a rural community of Delhi. Int J Med Sci Public Health 2015;4:298-303

Source of Support: Nil, Conflict of Interest: None declared. 